\title{
Pictures and Pedagogy: the role of diagrams in Feynman's early lectures, 1949-50
}

\section{By: Ari Gross}

Over the past decade historians and philosophers of science have developed an interest in Feynman diagrams, the simple, yet immensely powerful visual representations of subatomic events developed by Richard Feynman in the 1940s. Historical works like David Kaiser's Drawing Theories Apart and Adrian Wüthrich's recent doctoral thesis Feynman's Struggle and Dyson's Surprise Revelation (not to mention Sam Schweber's older work on the subject) have described how Feynman diagrams were developed and propagated. Philosophers such as James Brown and Letitia Meynell have discussed the question of what, precisely, Feynman diagrams represent. However, despite all the recent work contextualizing Feynman diagrams within Feynman's theoretical struggles, the post-doctoral and international academic systems, and the philosophical questions surrounding representation, few scholars have viewed Feynman diagrams as one of many visual techniques produced by Richard Feynman. Despite the excellent nature of many of these works, the majority of scholars who have addressed Feynman's diagrams have been seeing the forest for the trees: by focusing on "Feynman diagrams" at the expense of the numerous other types of diagrams used by Feynman, debates over the role of diagrams in scientific thought and the nature of visual reasoning have been unnecessarily narrowed.

This paper aims to step back and address the plurality of diagrams produced by Feynman, "Feynman diagrams" included, within their historical and textual context. In particular, I shall give a substantive account of how Feynman used diagrams in the first lectures in which he was tasked with explaining his new approach to quantum electrodynamics, those delivered at the University of Michigan in Ann Arbor and at the California Institute of Technology (Caltech), in 1949 and 1950, respectively. By critically examining unpublished course notes to two lecture series (which, to my knowledge, have not yet been discussed by historians and philosophers of science), Feynman's use and interpretation of diagrams, both "Feynman diagrams" and otherwise, will be illuminated.

While Feynman's early lectures contained a wide spectrum of diagrams employed in a multitude of ways, a hidden structure underlies his pedagogical use of images. The diverse forms of diagrams becomes comprehensible once each image is understood within its textual context, namely, the pedagogical point underlying its production. While each diagram was crafted to meet a specific, local need, we shall nevertheless be able to characterize several general themes regarding their use: diagrams could be used heuristically (as a conceptual aid to physical principles of his theory), computationally (as a mathematical tool), or as a heuristic aid to understanding the mathematical details of his approach.

While it is clear that Feynman modified each individual diagram to suit particular needs within the context of his lectures, a general visual style nevertheless persisted. This style, reminiscent of Minkowski space-time diagrams familiar to his audience, allowed him to move between morphologically distinct, yet stylistically similar, diagrams with ease. In doing so, Feynman 
employed his audiences' ability to reason and understand images analogically to facilitate the transmission of both general concepts and mathematical minutia relevant to his "new approach" to quantum electrodynamics. In examining how Feynman used such similar, yet distinct, diagrams through these lectures, we shall gain insight into nature of visual and analogical reasoning, subjects frequently discussed by historians and philosophers of science with a particular interest in cognitive science, such as Nancy Nersessian and the late David Gooding.

Furthermore, by situating these lectures within the context of previous and subsequent diagrams drawn by Feynman, we gain insight into the formation and establishment of scientific tools. When compared with Feynman's early diagrams, drawn in the years previous to these lectures (which were highly variable and rather ad hoc), and his increasingly widespread adoption of "Feynman diagrams" in later years, these lectures can be viewed as a "middle period", an era in which Feynman diagrams were present, but whose role was severely limited (though whether this was primarily due to Feynman's audiences' limited familiarity with Feynman diagrams and not to Feynman's latter recognition of the multitude of ways in which these diagrams could be used is a matter of debate). The insight gained into how Feynman diagrams were transformed from a purely computational device to a generalized (and, according to Kaiser, quite malleable) tool for scientific reasoning, synonymous with subatomic interactions themselves, particularly within their early context of being simply one of many types of diagrams, is quite valuable, as it can help in understanding the historical formation of standards within scientific practice.

Due to time constraints, it is unlikely that the totality of this paper will be able to be presented at \&HPS3. Regardless of which sections will be discussed, the importance of primary-source historical research in providing both stand-alone histories of science and critical insight into philosophical questions will be stressed. My talk will hopefully provide others with an example of how serious historical research can be used to address contemporary philosophical issues, such as visual and analogical reasoning and the formation and mutation of theoretical scientific tools, and how philosophical inquiries can, in turn, guide historical investigations. 